\title{
Comment on "Emergence of a Superconducting State from an Antiferromagnetic Phase in Single Crystals of the Heavy Fermion Compound $\mathrm{Ce}_{2} \mathrm{PdIn}_{8} "$
}

\author{
K. Uhlî́rová. . J. Prokleška, and V. Sechovský \\ Charles University, DCMP, Ke Karlovu 5, Praha 2, 121 16, Czech Republic
}

A recently published Letter [1] has reported on the antiferromagnetism (AF) and ambient-pressure superconductivity (SC) in a $\mathrm{Ce}_{2} \mathrm{PdIn}_{8}$ single crystal with $T_{\mathrm{N}} \sim 10 \mathrm{~K}$ and $T_{\mathrm{c}}=0.68 \mathrm{~K}$, respectively. Although we very much appreciate the effort exerted to prepare and characterize this new heavy fermion (HF) superconductor (SC), we would like to add a cautionary note that the reported Néel temperature coincides remarkably with $T_{\mathrm{N}}=10.2 \mathrm{~K}$ of $\mathrm{CeIn}_{3}$ [2]. It therefore leads us to considers the possible presence of $\mathrm{CeIn}_{3}$ in the samples that were investigated. In other $\mathrm{Ce}_{n} T \operatorname{In}_{3 n+2}(n=1,2)$ compounds [3 5] the AF is either absent $(T=\mathrm{Co}, \mathrm{Ir})$ or remarkably limited to much lower temperatures $(T=\mathrm{Rh})$. These compounds form a quasi-two-dimensional tetragonal structure with the $\mathrm{CeIn}_{3}$ and $T \mathrm{In}_{2}$ layers alternating along the (001) direction. Hence one might expect that the AF correlations develop within the $\mathrm{CeIn}_{3}$ layers while the interaction between the layers will be weaker as reported for $\mathrm{CeRhIn}_{5}$, an incommensurate $\mathrm{AF}$ $\left(T_{\mathrm{N}}=3.8 \mathrm{~K}\right)$ 6]. The remarkable agreement of the $T_{\mathrm{N}}$ values in the reported $\mathrm{Ce}_{2} \mathrm{PdIn}_{8}$ with the well-known $\mathrm{CeIn}_{3}$ is not discussed in the Letter [1]. Neither the striking discrepancy between their own results on single crystals [1] and polycrystals (reported paramagnetic down to $0.35 \mathrm{~K}[7$ ) has been explained. The absence of SC in the polycrystalline sample is explained by an unconventional coupling sensitive to structural disorder, internal strains, and/or tiny changes in the composition, but the disagreement in the magnetic ground state is not discussed at all.

Although a detailed phase analysis (X-ray diffraction and micro-probe) of the crystals was claimed to have been done [1, we would, however, still like to suggest that a $\mathrm{CeIn}_{3}$ single crystal covered by a single-crystalline layer of $\mathrm{Ce}_{2} \mathrm{PdIn}_{8}$ was in fact that which was investigated. From the reported heat capacity data, we estimate the amount of $\mathrm{CeIn}_{3}$ to be $15-20 \%$. In such case, a microprobe analysis of the sample's surface would not be able to detect it. Also, most of the diffraction peaks of both compounds interfere, because they have an almost equal lattice parameter $a=0.4693 \mathrm{~nm}$ [9] and $a=0.4689 \mathrm{~nm}\left[8\right.$ for $\mathrm{Ce}_{2} \mathrm{PdIn}_{8}$ and $\mathrm{CeIn}_{3}$, respectively.

Our first magnetization data obtained on crystals grown analogously to 11 were in agreement with the Letter. A careful microprobe analysis, however, indicated a presence of $\mathrm{CeIn}_{3}$, and element mapping showed that $\mathrm{Ce}_{2} \mathrm{PdIn}_{8}$ and $\mathrm{CeIn}_{3}$ form a sandwich-like system with well-defined regions (see Fig,1).

To confirm that the AF originates in $\mathrm{CeIn}_{3}$, we have measured more than 5 different $\mathrm{CeIn}_{3}$-free samples of $\mathrm{Ce}_{2} \mathrm{PdIn}_{8}$ by means of resistivity, heat capacity and magnetic susceptibility, and paramagnetic behaviour with significant magnetocrystalline anisotropy was observed down to a SC temperature [11.

The $\mathrm{SC}$ with $T_{\mathrm{c}}=0.7-0.45 \mathrm{~K}$ (sample dependent) has been confirmed in our samples. The difference of critical temperature is probably given by structural planar defects, which were also observed in $\mathrm{Ce}_{2} \mathrm{RhIn}_{8}$ [10. In agreement with 1, the $\mathrm{SC}$ has a HF character and it is a bulk property of the compound but it does not emerge out of a long-range AF state below the Néel temperature of $10 \mathrm{~K}$ because the reported $\mathrm{AF}$ was due to presence of an impurity phase.

* Electronic address: klara@mag.mff.cuni.cz

[1] D. Kaczorowski et al., Phys. Rev. Lett. 103, 027003 (2009).

[2] J. M. Lawrence et al., Phys. Rev. B 22, 4379 (1980).

[3] G. F. Chen et al., J. Phys. Soc. Jpn 71, 2836 (2002).

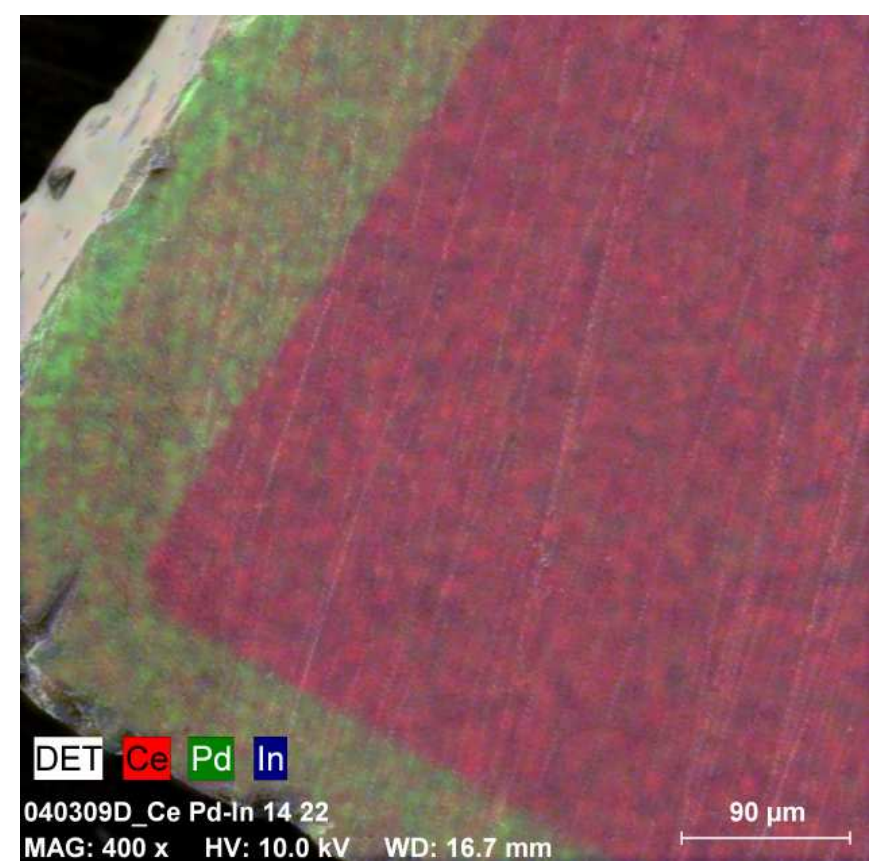

FIG. 1: EDX element mapping (Ce-red, Pd-green, In-blue) of a typical polished sample. $\mathrm{CeIn}_{3}$ (red region) is covered by a layer of $\mathrm{Ce}_{2} \mathrm{PdIn}_{8}$ and $\mathrm{Ce}_{1.5} \mathrm{Pd}_{1.5} \mathrm{In}_{7}$ (thin dark green). 
[4] J. S. Kim et al., Phys. Rev. B 69, 024402 (2004).

[5] R. Movshovich et al., Phys. Rev. Lett. 86, 5152 (2001).

[6] W. Bao et al., Phys. Rev. B 62, R14621 (2000).

[7] D. Kaczorowski et al., Phys. B 404, 2975 (2009).

[8] I. R. Harris et al., J. Less Comm. Met. 9, 7 (1965).

[9] D. V. Shtepa et al., Mow. Univ. Chem. Bull. 63, 162
(2008).

[10] E. G. Moshopoulou et al., Acta Crystallogr. Sect. B 62, 173 (2006).

[11] K. Uhlířová et al., Intermetallics , To Be Published (2010). 